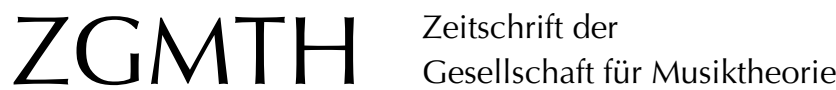

Scheideler, Ullrich (2015): Musikalische Zeitgestaltung in Mendelssohns Liedern ohne Worte. ZGMTH 12/2, 223-243. https://doi.org/10.31751/820

\section{(c) 2015 Ullrich Scheideler}

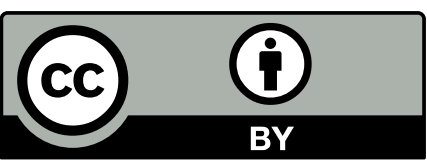

Dieser Text erscheint im Open Access und ist lizenziert unter einer Creative Commons Namensnennung 4.0 International Lizenz.

This is an open access article licensed under a

Creative Commons Attribution 4.0 International License.

veröffentlicht / first published: 30/07/2016 zuletzt geändert / last updated: 18/03/2017 


\title{
Musikalische Zeitgestaltung in Mendelssohns Liedern ohne Worte
}

\author{
Ullrich Scheideler
}

ABSTRACT: Felix Mendelssohn Bartholdys Liedern ohne Worte liegt gewöhnlich eine einfache dreiteilige Liedform mit überschaubaren Proportionen zugrunde. Anhand dreier Punkte im Formverlauf (Themenvorstellung im ersten Formteil, Übergang zur Reprise, Themenreprise) wird zu zeigen versucht, wie durch Erweiterung der achttaktigen Einheiten sowie die besondere Gestaltung der Harmonik und Motivik dieses Formgerüst aufgebrochen und gleichsam dynamisiert wird. Dabei wird insbesondere das Ineinander von Andeuten und (vorläufigem) Aufschieben eines (harmonischen, thematischen) Ziels als Sehnsuchtston und damit im Sinne einer romantischen Ästhetik gedeutet.

Felix Mendelssohn Bartholdy's Songs without words are generally written in a simple ternary form with straightforward proportions. Three points in the course of the piece (the presentation of the theme in the first part, the transition to the recapitulation, and the recapitulation of the theme) serve to illustrate how the extension of the eight-bar-units as well as the special design of harmonic and motivic processes open up and dynamize this formal framework. The interaction of adumbration and a (provisional) deferral of the harmonic and thematic goal is interpreted as a note of longing and thus in terms of a romantic aesthetic.

Felix Mendelssohn Bartholdys Lieder ohne Worte, zwischen 1832 und 1845 in sechs Heften zu je sechs Stücken erschienen', gelten gemeinhin als unkomplizierte, gefällige Stücke, die kaum der Analyse lohnen. ${ }^{2}$ Die Eleganz der musikalischen Oberfläche, die einfache Satztechnik wie die formale Einfachheit einer fast immer realisierten dreiteiligen Liedform verdecken freilich die Tatsache, dass sich anhand dieser Stücke einige grundlegende kompositorische Möglichkeiten musikalischer Zeitgestaltung studieren lassen.

Betrachtet man die Stücke unter dem Aspekt einer Problemgeschichte des Komponierens, so kann man in einer ersten Annäherung festhalten, dass Strategien zu beobachten sind, die Taktgruppengliederung der (engen und strengen) musikalischen Form so aufzubrechen, dass im Inneren individuelle Zeitverläufe möglich wurden. Anders als bei Robert Schumann oder Frédéric Chopin, die häufig vor allem durch eine »romantische Harmonik $\ll^{3}$, durch besondere Spielfiguren oder ein spezifisches Zusammenwirken von

1 Postum erschienen zwei weitere Sammlungen, sodass ungefähr 50 Stücke vorliegen.

2 Das geht bereits daraus hervor, dass es im Unterschied zu den Klavierwerken Robert Schumanns zu den Liedern ohne Worte Felix Mendelssohn Bartholdys kaum analytische Literatur gibt.

3 Vgl. Moßburger 2005. 
Melodik, Harmonik und Kontrapunkt bei gleichzeitiger Beibehaltung von achttaktigen Einheiten einen individuellen romantischen Ton in ihrer Musik anzuschlagen versuchten, lässt sich bei den Liedern ohne Worte die Idee verfolgen, die Form selbst als wesentliches Moment der Individualisierung heranzuziehen. Denn die dreiteilige Liedform wird oft nur noch als Hintergrundfolie verwendet und durch verschiedene Maßnahmen vor allem an ihren Abschnittsgrenzen gleichsam verflüssigt. Wie die nachfolgenden Analysen zu zeigen versuchen, kommt es dadurch zu einer Dynamisierung der Form: Das Formgerüst ist nun nicht mehr etwas Blockhaftes oder Statisches, sondern gerät in Bewegung. Damit ist auch die Vorhersehbarkeit des musikalischen Verlaufs sowohl im Hinblick auf seine zeitliche Ausdehnung als auch in Bezug auf den musikalischen sInhalt nicht mehr gewährleistet.

Im Folgenden sollen zunächst die in der Musiktheorie des 18. und frühen 19. Jahrhunderts erörterten Möglichkeiten, einfache syntaktische Gebilde bzw. symmetrisch gebaute Themen zu verändern (d. h. vor allem zu erweitern), knapp rekapituliert werden. Anschließend soll dann ein Menuett von Joseph Haydn als typisches Beispiel für den Einsatz von melodischen Verlängerungsmitteln in der zweiten Hälfte des 18. Jahrhunderts analysiert werden, um vor diesem Hintergrund das Besondere des Verfahrens bei Mendelssohn zu erfassen. Abschließend soll versucht werden, den Zusammenhang von technischem Verfahren und romantischem Ton genauer zu bestimmen.

Die Verfahren, ein achttaktiges musikalisches Gebilde zu erweitern (seltener auch zu

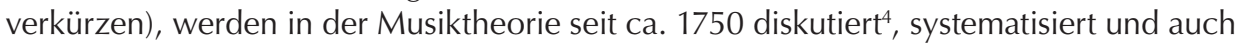
in Kompositionslehren des frühen 19. Jahrhunderts bisweilen ausführlich erörtert, ohne dass es hier zu grundlegenden Änderungen kam. Grundsätzlich wird dabei zwischen Erweiterungen im Inneren einer Taktgruppe und solchen am Ende unterschieden (Adolf Bernhard Marx führt zudem äußere Erweiterungen am Beginn ein ${ }^{5}$ ).

Joseph Riepel differenziert kurz nach 1750 bei Erweiterungen im Inneren zwischen der Wiederholung (meist eines Zweitakters) und dem sogenannten »Einschiebsel $\aleph^{6}$, mit dem er die Einfügung einer Taktgruppe bezeichnet, deren Melodik sich von den umliegenden Takten deutlich abhebt. Außerdem kennt er noch die »Ausdehnung ${ }^{7}$, die nicht durch zusätzliches thematisches Material, sondern in der Regel durch Augmentation von Notenwerten zustande kommt. Die Hinzufügung von Takten am Ende wird »Verdoppelung der Kadenz $«^{8}$ genannt. Eine Generation später hat Heinrich Christoph Koch den Strategien der Erweiterung vor allem im 3. Band seiner Kompositionslehre ein umfangreiches Kapitel gewidmet, das er „Von dem Gebrauche der melodischen

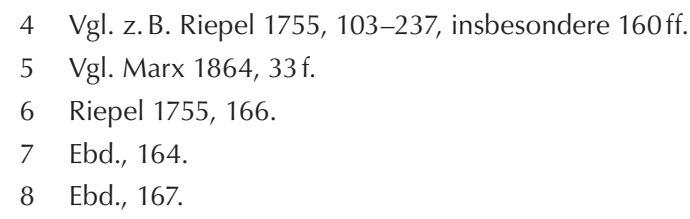


Verlängerungsmittel ${ }^{9}$ überschrieben hat. Seine Systematik ist zwar differenzierter als die Riepels, listet aber im Wesentlichen dieselben Mittel auf, einen größeren Umfang zu erreichen. Er benennt ebenfalls die Wiederholung, das »Einschiebsel« (als »Einschaltung zufälliger melodischer Theile $«^{10}$ bzw. »Ausdehnung ${ }^{11}$ und als »Parenthese $«^{12}$ bezeichnet), bespricht zudem ausführlich die Möglichkeit der Fortführung durch Sequenzen sowie einer Technik, die man unter dem Begriff der motivischen Arbeit mittels Abspaltung zusammenfassen könnte. ${ }^{13}$ Die Hinzufügung am Ende wird als »Vervielfältigung der Absatzformeln und Cadenzen $\aleph^{14}$ bezeichnet.

Dass die Ideen von Riepel und Koch auch in der ersten Hälfte des 19. Jahrhunderts noch aktuell waren, zeigt der zweite Band der Kompositionslehre von Adolf Bernhard Marx, die als wesentliche Kategorie kompositorischen Denkens die Motivik ins Zentrum rückt. Hier werden die Erweiterungsmöglichkeiten erstmals nach der Art, wie ein Thema motivisch gegliedert ist, mithin gemäß »Satzform« und »Periode», differenziert. Die Satzform lässt fünf Möglichkeiten zu: die »Ausdehnung der Schlussmomente ${ }^{15}$, die ${ }_{\text {Wieder- }}$ holung der Schlussformel $\ll^{16}$, die "Wiederholung der letzten Motive $«^{17}$, die Einleitung $«^{18}$, schließlich "gangartige Sätze «. ${ }^{19}$ Die Besonderheit der Satzform bedingt es, dass vier der fünf genannten Punkte sich auf das Ende oder den Anfang beziehen. Nur die gangartigen Sätze führen zu einer Erweiterung im Inneren. Bei der Periode nimmt Marx hingegen von Beginn an eine Unterscheidung in »äußere Erweiterung « und »innere Erweiterung « ${ }^{20}$ vor. Äußere Erweiterung sind für ihn die Ausdehnung des Schlussakkords, die Einleitung, schließlich der »Anhang «" ${ }^{21}$. Was bei Koch bereits anklang ${ }^{22}$, wird bei Marx in diesem Zusammenhang deutlicher hervorgehoben: Will man eine Kadenz verdoppeln bzw. die Schlussformel wiederholen, so muss (oder soll) der erste Schluss unvollkommen sein, oder in den Worten von Marx: „Wir müssen also [...] die Kraft des Schlusses brechen, das heisst, ihn in irgendeinem Punkte zu einem unvollkommenen und darum weniger befriedigenden machen «. ${ }^{23}$ Es gibt hierfür drei grundsätzliche Möglichkeiten: den Einsatz einer anderen Stimme unmittelbar nach Erreichen des Schlussklangs, die »Störung der Harmo-

9 Koch 1793, 153-231. Erweiterungen werden zudem bereits in Theil II (1787) im Abschnitt »Von der Beschaffenheit der melodischen Theile» diskutiert.

10 Ebd., 218.

11 Ebd., 164.

12 Ebd., 218.

13 Vgl. ebd., $210 \mathrm{ff}$.

14 Ebd., 191.

15 Marx 1864, 27.

16 Ebd., 29.

17 Ebd., 31.

18 Ebd., 33.

19 Ebd., 34.

20 Ebd., $41 \mathrm{ff} ., 46 \mathrm{ff}$.

21 Ebd., 43.

22 Vgl. Koch 1793, 194.

23 Marx 1864, 44. 
nie des Schlusses ${ }^{24}$ dadurch, dass die Schlusstonika nicht in Oktavlage oder in Grundstellung erklingt, schließlich den Trugschluss. Unter innerer Erweiterung fasst Marx zunächst die (verzierte) Wiederholung von Vorder- oder Nachsatz, sodass ein 12-Takter bzw. eine Periode zu drei Sätzen entsteht, sowie ein 16-taktiges Thema, das sich aus jeweils erweitertem Vorder- und Nachsatz zusammensetzt. Andere Formen werden unter der Überschrift »Weitere und freiere Bildungen $\aleph^{25}$ abgehandelt. Hier fällt auch Riepels Begriff des »Einschiebsel[s] $\ll^{26}$, das nun einen Zweitakter zwischen Vorder- und Nachsatz bezeichnet.

Mit Ausnahme der Verdoppelung der Kadenz, die bei allen drei Theoretikern als Mittel der Verstärkung der Schlusswirkung eingeführt wird, bleiben die Aussagen darüber, welcher musikalische (bzw. formale) Zweck mit der Erweiterung verbunden sein kann, eher vage. Koch fordert dazu auf, »den vortheilhaftesten Gebrauch derselben den Meistern der Kunst durch fleißiges Studium ihrer Werke abzulernen zu suchen ${ }^{27}$, da »sich nicht allgemein bestimmen« lasse, »[w]enn, und wo jedes dieser Hilfsmittel [...] gebraucht werden ${ }^{28}$ könne. Als allgemeines Kriterium gibt er allerdings an, dass es bei einer Wiederholung eines melodischen Teils auf die Deutlichkeit der Empfindung ankomme. ${ }^{29}$ Auch Marx konzentriert sich vornehmlich auf technische Mittel. Gemäß seinen kompositorischen Schlüsselkategorien geht er vom Motiv aus und fordert, dass eine Erweiterung nur dann stattfinden könne, wenn erstens das Motiv »Interesse wecken « ${ }^{30}$ könne und es zweitens dann zu einer "folgerechten Entwicklung « ${ }^{31}$ komme. Dabei sei darauf zu achten, dass eine Mitte zwischen reiner Wiederholung des Motivs und immer neuen Wendungen gewahrt bleibe.

Im Zusammenhang mit der Beschreibung und Klassifizierung von Erweiterungen bzw. allgemeiner von Formverläufen können schließlich auch die Kategorien herangezogen werden, die Kofi Agawu in seinem 1991 erschienenen Buch Playing with Signs entwickelt hat. ${ }^{32}$ Agawus der Semiotik verpflichteter Ansatz unterscheidet zwischen »extroversive semiosis $^{33}$, die grob als Anwendung von Topoi oder sreferential signs« umschrieben werden $\mathrm{kann}^{34}$, und »introversive semiosis $\aleph^{35}$, die sich auf die Form bezieht und als

24 Ebd.

25 Ebd., $50 \mathrm{ff}$.

26 Ebd., 51.

27 Koch 1793, 156.

28 Ebd.

29 Vgl. ebd., 155: „Allein der angehende Tonsetzer, wenn er sich dieses Mittels bey seinen Ausarbeitungen bedient, darf dabey nicht außer Acht lassen, daß ein melodischer Theil, welcher der Wiederholung würdig seyn soll, entweder schon einen hohen Grad der Darstellung der auszudrückenden Empfindung enthalten, oder daß man demselben bey der Wiederholung neuen Stoff zum Ausdrucke der Empfindung zu geben suchen muß.«

30 Marx 1864, 47.

31 Ebd., 57.

32 Vgl. Agawu 1991.

33 Ebd., $26 \mathrm{ff}$.

34 Eine Liste solcher referential signs` findet sich ebd., 30. Es handelt sich dabei um sehr verschiedene

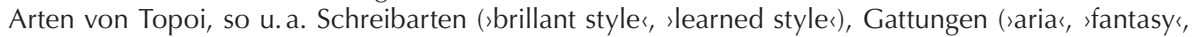
Opera buffa), Tanzsätze (Bourrée, Gavotte), Charakterisierungen, die sich auf Regionen oder Länder 
»beginning-middle-end paradigm « oder auch als Anwendung von spure signs bezeichnet wird. ${ }^{36}$ >Anfang`, >Mitte und `Ende` zeichnen sich durch eine jeweils spezifische Art des musikalischen Verhaltens (im Hinblick auf Harmonik, Motivik, übergeordnete Bewegung, Außenstimmensatz etc.) aus und können zugleich auf verschiedenen Ebenen angesiedelt werden. Nicht nur ein gesamtes Stück lässt sich mit diesen Kategorien umschreiben, sondern auch einzelne Formteile oder gar Abschnitte, sodass auch von einem `Anfang vom Ende` etc. gesprochen werden kann. Derartige sstructural signs` lassen sich gleichsam als Markierungen oder Wegweiser interpretieren, die dem Hörer den formalen Sinn einer Stelle aufschlüsseln. Für die Untersuchung der Zeitgestaltung können solche Kategorien dann herangezogen werden, wenn es darum geht, Proportionen genauer zu erfassen (etwa als Raffung oder Dehnung) oder das Verhältnis von eigentlichem formalen Sinn und konkreter Realisierung genauer zu bestimmen.

Erweiterungen sind im späten 18. Jahrhundert nicht zuletzt im Menuett oder Scherzo Standard. Wie man zumindest für viele Menuette Haydns zeigen kann, werden die unterschiedlichen Arten der Erweiterungen dazu genutzt, um ein »Playing with signs « in Gang zu setzen. Die achttaktigen Modelle wie die Form im Großen erlauben dabei die Identifizierung einer Taktgruppe als einen Anfang, eine Mitte oder ein Ende (nach den Kategorien von Kofi Agawu handelt es sich hierbei um sintroversive signs`). Damit stehen Erwartungen des Hörers im Hinblick auf den Fortgang im Raum, die sich bewusst in das kompositorische Kalkül einbeziehen lassen. Genau dies tut Haydn, und zwar auf eine Art, die diese Erwartungen oft mehrfach konterkariert. Als Beispiel sei hier das Menuett aus der Sinfonie Nr. 35 (B-Dur; 1767) herangezogen: Der A-Teil umfasst zehn Takte (vgl. Bsp. 1). Nach einem regulären Vordersatz (T. 1-4) beginnt der Nachsatz mit der Wiederholung der Phrase, doch wird diese nicht unmittelbar in eine Ganzschlusskadenz fortgeführt. Vielmehr wird der musikalische Verlauf plötzlich und unerwartet durch ein »Einschiebsel« mit neuer Motivik und unter Aussparung des Basses unterbrochen. Erst danach wird die Periode fortgesetzt bzw. zum Abschluss gebracht. Aus dieser inneren Erweiterung im A-Teil zieht Haydn nun im A'-Teil überraschende Konsequenzen (vgl. Bsp. 2). Denn nun erklingt das Thema als Achttakter mit regulärem vollkommenem Ganzschluss ohne das Einschiebsel. Doch ist die Periode damit noch nicht an ihr Ende gelangt: Das vormalige Einschiebsel taucht nun unerwartet als Anhang wieder auf, initiiert dann einen zweiten Zweitakter (im Forte mit der Unisono-Satztechnik der Phrase von Takt 1-2, aber in Anlehnung an die Motivik der Kadenztakte 9-10), dem ein weiterer Zweitakter als Kadenz wie im A-Teil folgt. Der A'-Teil zeigt mithin ein (unterhaltsames) Spiel mit Versatzstücken, v. a. mit Segmentierungen und Neuzuordnungen einzelner Parameter wie Motivik oder Instrumentierung im Zusammenhang der Neugruppierung der Taktgruppen (das betrifft hier auch die Satztechnik, so erklingt die Wiederholung der Phrase im Nachsatz des A'-Teils jetzt nicht im Unisono, sondern als homophoner Satz, mithin in der Satztechnik der Gegenphrase).

(`Turkish music`), auf funktionale Musik ('fanfare`, shunt style`, smarch`) oder auf im weitesten Sinne stilistische Merkmale (Empfindsamkeit, Sturm und Drang) beziehen. In seinem 2009 erschienenen Buch Music as Discourse, das sich primär auf die Musik des 19. Jahrhunderts bezieht, hat Agawu die Liste der Topoi noch erweitert (vgl. Agawu 2009, 43 f.).

35 Agawu 1991, $51 \mathrm{ff}$. 


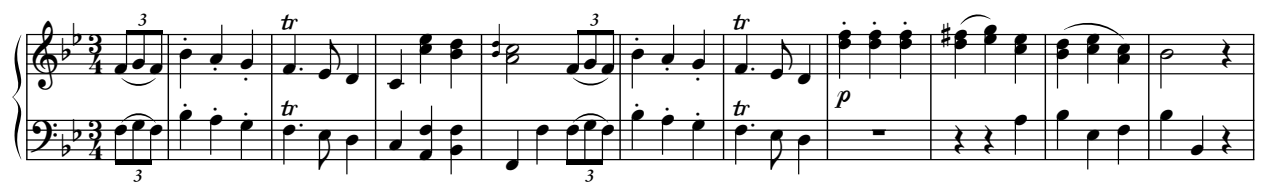

Beispiel 1: Joseph Haydn, Sinfonie Nr. 35, Menuett, T. 1-10
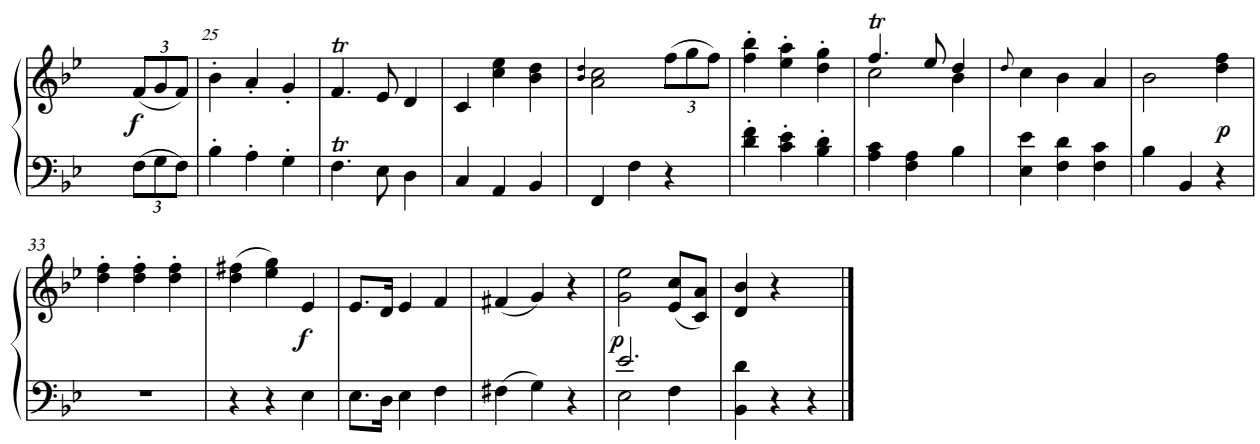

Beispiel 2: Joseph Haydn, Sinfonie Nr. 35, Menuett, T. 25-38

II

In Mendelssohns Liedern ohne Worte sind in technischer Hinsicht ganz ähnliche Verfahren der melodischen Verlängerung, wie sie von den zuvor genannten Theoretikern des 18. und 19. Jahrhunderts beschrieben wurden, zu finden. Im Unterschied zu den Realisierungen etwa bei Haydn scheint mit ihnen indes ein anderer musikalischer Sinn verbunden zu sein. Die verschiedenen Formen der Erweiterungen lassen sich zwar auch als ein »Playing with signs« interpretieren, doch stehen diese ganz im Dienst einer Strategie, den Stücken einen besonderen Ton zu verleihen, den man vielleicht am besten als >romantischen Sehnsuchtston Idee besteht dabei darin, dass ein bestimmtes musikalisches Ziel zwar früh musikalisch erkennbar wird, das Erreichen dieses Ziels aber (mehrfach) aufgeschoben wird. Die einfache dreiteilige Liedform wie die spezifische Ausfüllung lassen zwar das Ziel mehr oder weniger deutlich aufscheinen, aber zugleich das (vorläufige) Nicht-Erreichen wie das (sehnsuchtsvolle) Bemühen gleichsam um Erfüllung fühlbar werden. Diesem Ineinander von anvisiertem Ziel und vorläufigem Nichterreichen soll im Folgenden anhand dreier Stationen im Formverlauf nachgegangen werden: erstens bei der Themenvorstellung im ersten Formteil, zweitens beim Übergang zur Reprise nach einem kontrastierenden Mittelteil, schließlich drittens in der Themenreprise. Der letzte Punkt soll anhand einer knappen Analyse zweier vollständiger Stücke veranschaulicht werden.

36 Ebd. 


\section{Themenvorstellung im ersten Formteil}

Typisch für den Bau der Themen im A-Teil der Lieder ohne Worte ist eine Syntax mit klarer Gliederung in Vordersatz und Nachsatz, welche sich in der Regel auf ein achttaktiges oder 16-taktiges Modell bezieht und als Periode oder Satz beschreibbar ist. Allerdings erklingt das Modell fast nie in seiner reinen Form, sondern wird meist verändert bzw. erweitert. Dabei umfasst der Vordersatz fast immer ganz regelmäßig vier oder acht Takte und macht somit das Modell als Referenzpunkt für den Hörer klar; der Nachsatz ist hingegen erweitert. Nach den Kategorien von Riepel, Koch und Marx lassen sich die meisten zusätzlichen Takte als Verdoppelung der Kadenz oder äußere Erweiterung interpretieren. Wie in den theoretischen Schriften beschrieben, hat dies eine Bekräftigung der Schlusswirkung zur Folge: Das Ende des ersten Formteils wird auf diese Weise sinnfällig gemacht, die Kadenzverdopplung hat also formale Funktion. Mendelssohn nutzt diese Bekräftigung des Schlusses jedoch gleichzeitig dazu, um das Kontinuum der gleichmäßig abrollenden Zeit zu durchbrechen. Die Betrachtung dreier Werkausschnitte soll diese These untermauern und verschiedene Spielarten vorführen.

Im Lied ohne Worte Es-Dur (MWV U 109; vgl. Bsp. 3), das als zweites Stück in der Sammlung op. 53 veröffentlicht wurde, erstreckt sich das Thema über 20 Takte. Es ist periodisch gebaut und besteht aus jeweils viertaktigen Phrasen und Gegenphrasen. Nach einem >Vortaktı (T. 1) endet der Vordersatz regulär in Takt 9 halbschlüssig, der Nachsatz entspricht sechs Takte lang dem Vordersatz und mündet ab Takt 16 in die Kadenztakte, die in Takt 17 in die Tonika Es-Dur in Grundstellung und Oktavlage hätten führen können. Dies geschieht jedoch nicht. Stattdessen springt die Melodie in Takt 17 in den Quintton $b^{1}$ und in einen Es-Dur-Sextakkord (eingeleitet durch die Septime as im Bass in T. 16). Die Schlusskadenz ist damit aufgeschoben, sodass ein zweiter Anlauf erfolgen muss. In einer einfachen Variante hätte sich ein Zweitakter anschließen können, der ungefähr den Kadenztakten 20 (mit Auftakt) und 21 entsprochen hätte. Stattdessen folgen vier Takte, die mehrere Besonderheiten aufweisen: Erstens sind die Zwischendominanten in Verbindung mit einer (unregelmäßigen) Terzfallsequenz auffällig, die sich vor dem Hintergrund eines zuvor wesentlich diatonischen Satzes umso stärker abheben. Zweitens kommt es in den Takten 17-19 zu einer Hemiolenbildung. Drittens ist mit diesem Abschnitt gleichsam eine Wiederholung des Themas in geraffter Form verbunden, und zwar sowohl in melodischer als auch harmonischer Hinsicht: Der Spitzenton $g^{2}$ wird nochmals angesprungen und zum Ausgangspunkt einer übergeordneten und über eine Oktave fallenden melodischen Bewegung, die in Takt 21 endlich es ${ }^{1}$ erreicht; die harmonische Bewegung über c-Moll, As-Dur, f-Moll und B-Dur vollzieht dabei den Verlauf der Takte 5 ff. bzw. 13 ff. noch einmal in Umrissen nach. Damit erhalten die Takte 17-21 einen doppelten Sinn: In formaler Hinsicht handelt es sich um eine Verdoppelung der Kadenz mit dem Ziel, die erreichte Formstation, also das Ende der Themenpräsentation bzw. des ersten Teils zu betonen. Mendelssohn nutzt nun diese Takte gleichzeitig dazu, die sinnere Zeits und den Charakter der Musik zu verändern. Wo zuvor eine diatonische Harmonik meist ganztaktig wechselte und ein hohes Gleichmaß der Bewegung vorlag, wird nun ein Moment der Komprimierung und damit der Intensivierung oder Beschleunigung erzeugt. Im Vorder- bzw. Nachsatz zeichneten sich die Phrasen durch 
einen Abstieg von $g^{2}$ bis $g^{1}$ aus (vgl. T. 3-5 bzw. T. 11-13), während die Gegenphrase auf dem $g^{1}$ verharrte und zu $f^{1}$ hinabstieg (vgl. T. 5-9 bzw. T. 13 ff.). Die Verdoppelung der Kadenz mit dem schließlich erreichten Grundton wird nun als eine Art Durchbruch inszeniert: Das Ziel $e s^{1}$ ist das Ergebnis eines von großer Emphase geprägten Verlaufs (bzw. zweiten Kadenzanlaufs), dem etwas Schwärmerisch-Übermütiges anhaftet. Das technische Moment der Kadenzverdopplung wird genutzt für einen besonderen musikalisch-poetischen Ton.
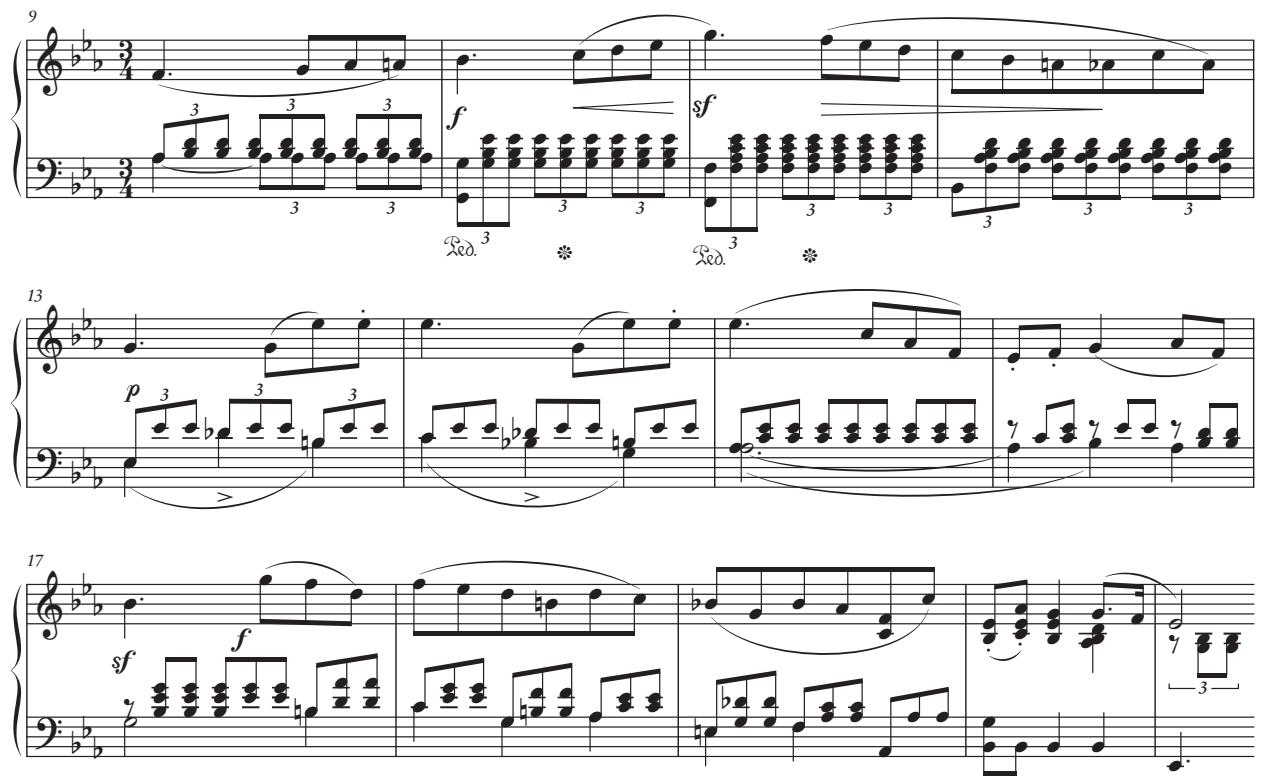

Beispiel 3: Felix Mendelssohn Bartholdy, Lied ohne Worte op. 53/2 (MWV U 109), T. 9-21

Ungleich aufwändiger ist die Verlängerung im Lied ohne Worte a-Moll op. 19/2 (MWV U 80; vgl. Bsp. 5), dessen Thema ebenfalls ein 16-taktiges Modell zugrunde liegt, das hier aber auf 29 Takte, also fast die doppelte Länge, gedehnt erscheint. Nachdem der reguläre achttaktige Vordersatz halbschlüssig endet, beginnt mit Takt 9 der Nachsatz, dessen bis Takt 12 reichende Phrase gegenüber den Takten 1-4 nur minimal verändert ist. Erst die Gegenphrase ab Takt 13 ist neu und dann deutlich erweitert. Für die Harmonik ist von Bedeutung, dass es in der Gegenphrase des Nachsatzes zu einer Ausweichung in die Oberquinttonart e-Moll kommt. Eine Besonderheit einer derartigen Ausweichung besteht darin, dass es nicht a priori eine obligate Lage für die Schlussnote der Melodie gibt: Ob das Ziel $e^{1}$ oder (eine Oktave höher) $e^{2}$ sein wird, steht nicht von vornherein fest. Genau diese Ambivalenz wird in Form eines Changierens der anvisierten Oktavlage von Mendelssohn ausgenutzt. Würde die Syntax des Themas das 16-taktige Modell erfüllen, so könnte in Takt 15-16 eine Kadenz erklingen, die mit den Akkorden H-Dur-e-Moll auf $e^{2}$ enden würde (vgl. Bsp. 4). 


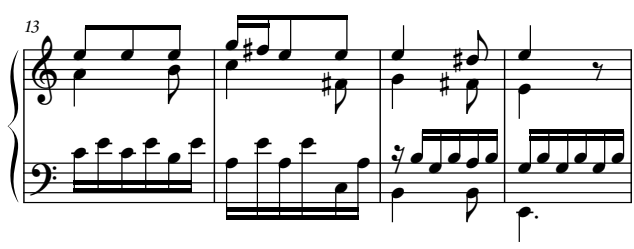

Beispiel 4: Felix Mendelssohn Bartholdy, Lied ohne Worte op. 19/2 (MWV U 80), hypothetische Version der T. 13-16

Eine derartige Kadenz ist jedoch nicht komponiert. Zunächst werden die Takte 13/14 wiederholt, sodass ein erster Kadenzanlauf erst auf die Takte 22/23 zielt: Die Melodie hat sich bereits bis $g^{1}$ gesenkt, in dem absteigenden Zug, der auf $e^{1}$ zielt, fehlen also nur noch die Töne fis ${ }^{1}-e^{1}$, um einen Ganzschluss in Oktavlage zu erreichen. Stattdessen geht die Melodie nach oben erst über $a^{1} z u h^{1}$, dann eine Oktave höher zu g $g^{2}$ mit Weiterführung zu fis ${ }^{2}$ und $\mathrm{e}^{2}$, das in Takt $23 \mathrm{f}$. als Quartvorhalt über dem Dominantgrundton $h$ erklingt, was wieder auf eine Kadenz - also den zweiten Kadenzanlauf - hindeutet. Die Erwartung einer Auflösung zum Leitton $d i s^{2}$ wird indes enttäuscht: $e^{2}$ geht zu $d^{2}$ in Verbindung mit einem G-Dur-Septakkord, der zu C-Dur führt (T. 25), womit eine Variante der in e-Moll trugschlüssigen Verbindung $\mathrm{H}^{7}-\mathrm{C}$ vorliegt. Das Ausbleiben des Leittons dis $^{2}$ leitet dann den Abwärtstrend der Melodie ein, sodass die Kadenz erst im dritten Anlauf ihren Abschluss findet, und zwar nun doch in der unteren Oktave (also auf $e^{1}$ ), die bereits in Takt 22/23 anvisiert worden war.

Die Verlängerung des Themas kommt hier also dadurch zustande, dass es nun gleich mehrere Kadenzanläufe braucht, um das Ziel e-Moll zu erreichen: Nachdem Mendelssohn die erste (reguläre) Kadenzmöglichkeit in Takt 16 hat verstreichen lassen, zielt die erste Kadenz auf Takt 22/23, die zweite auf Takt 25, erst die dritte in Takt 29 erreicht das Ziel. Dabei werden die Kadenzsignale vor allem dadurch immer deutlicher, dass die Dominante ein stärkeres Gewicht erhält (aufgrund der Länge und des Bassregisters). Hinzu kommt die Dehnung der Prädominante, die sich über sechs Takte (T. 13-18) erstreckt. Die Erweiterung des Nachsatzes kommt im Großen mithin durch zwei Prolongationen zustande: durch die der Prädominante (durch Verharren auf der Subdominante), dann die der Dominante (durch Trugschlüsse im weitesten Sinne bzw. Verdoppelung der Kadenzen). Verbunden sind diese Prolongationen mit einer Melodieführung, die durch viele Richtungswechsel und Sprünge immer unruhiger wird. Man kann mithin den Nachsatz als (zähen) Versuch interpretieren, ein Ende in der hohen Oktavlage zu finden, der aber nicht gelingt, sodass (resignierend) doch die untere Oktavlage als Ziel angesteuert und schließlich erreicht wird.

Während man für Opus 19/2 davon sprechen kann, dass ein Ziel zwar anvisiert, aber letztlich verfehlt wird, sodass zu einem anderen Ziel ausgewichen wird, passiert in Opus 19/1 (MWV U 86; vgl. Bsp. 6) genau das Umgekehrte. Hier ist das melodische Ziel früh unverkennbar, doch lässt etwas die Musik zögern, den Schritt zu diesem Ziel tatsächlich zu vollziehen. Auch hier ist der Vordersatz regulär, während der Nachsatz stark erweitert erscheint. Die erste Erweiterung kommt durch ein Stillstehen auf der Prädominante (bezogen auf die Oberquinttonart H-Dur) in den Takten 9/10 zustande, die erstmals mit einer Durchbrechung der Satztechnik einhergeht (Melodie und Bass antworten einander). Das Stehenbleiben (oder aber Zögern) wird erst durch den Übergang 

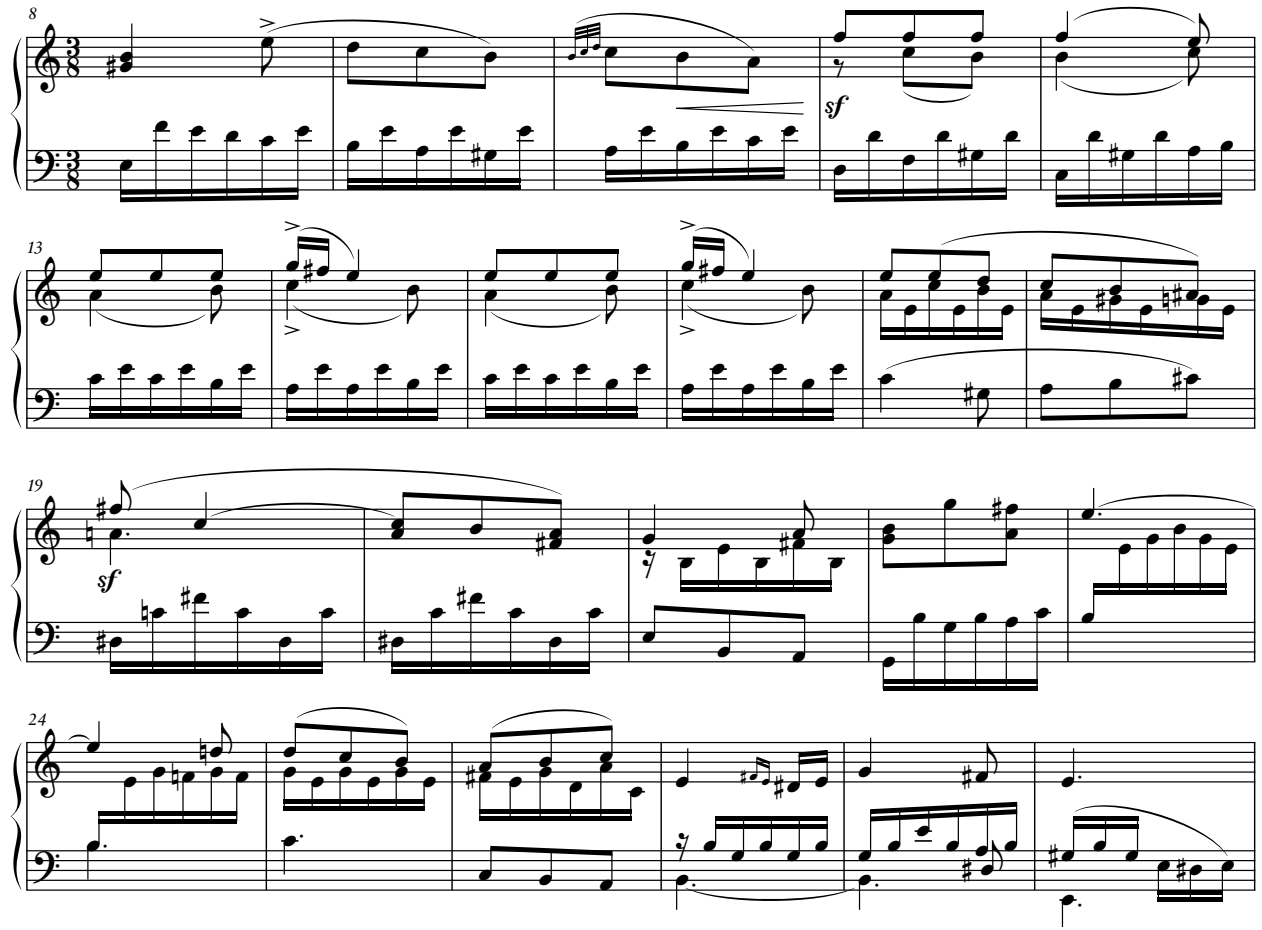

Beispiel 5: Felix Mendelssohn Bartholdy, Lied ohne Worte op. 19/2 (MWV U 80), T. 8-29
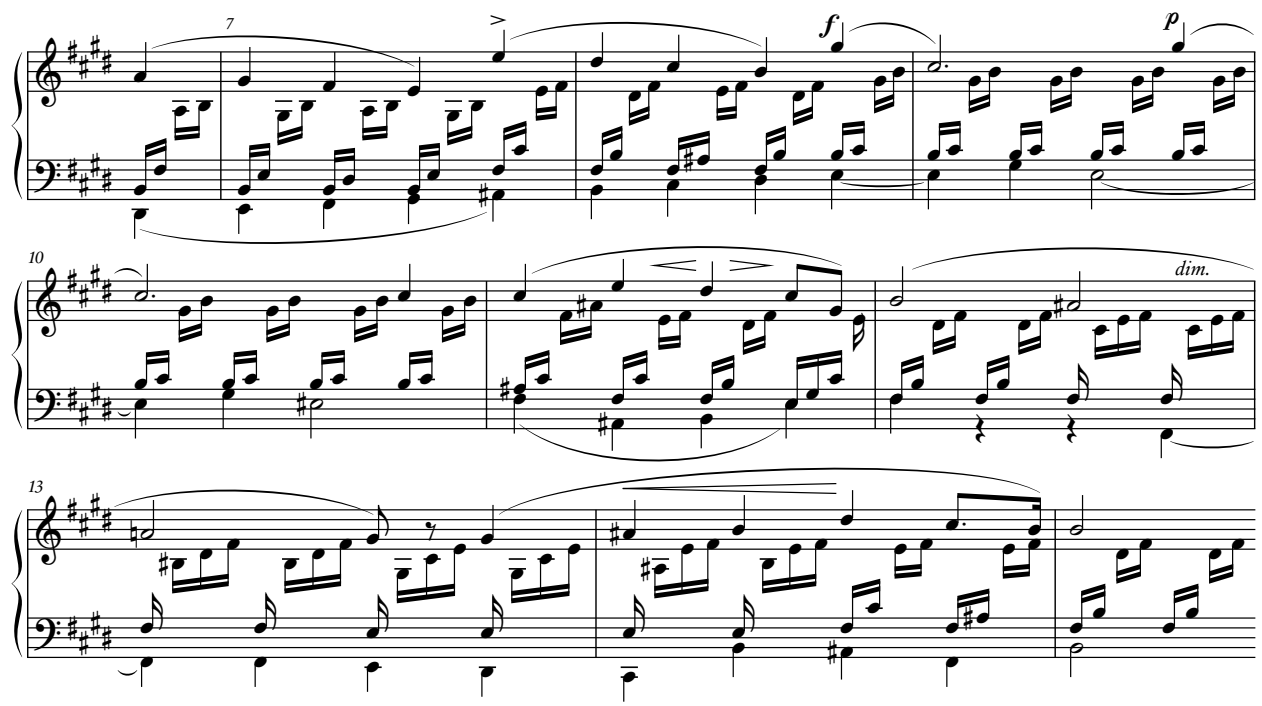

Beispiel 6: Felix Mendelssohn Bartholdy, Lied ohne Worte op. 19/1 (MWV U 86), T. 6-15 
zur Doppeldominante (zweite Hälfte von T. 10) beendet, doch vollzieht dann die in Takt 12 erreichte starke Dominante nicht den Übergang in die Tonika, sondern schweift noch einmal (über eine Zwischendominante) in die Subdominantparallele ab (zweite Hälfte T. 13). Erst die zweite Kadenz führt schließlich zum Ziel.

Alle drei Ausschnitte zeigen ein Hinausschieben der Schlusskadenz im A-Teil. Zunächst wird dadurch nichts anderes als eine größere Ausdehnung des eröffnenden Themas sowie die Betonung des Endes des Formteils `Themenpräsentation` erreicht. Entscheidend jedoch ist, dass durch die spezifische Gestaltung dieses Verfehlens des Ziels im ersten Anlauf die Themen selbst, ihre innere Zeitgestaltung und damit ihr Charakter eine zusätzliche Bestimmung erfahren. Während in Opus 53/2 das Thema zu etwas Übermütig-Rauschhaftem mutiert, ist in Opus 19/2 ein Moment der Anstrengung und in Opus 19/1 ein Moment des bewussten Hinausschiebens des Ziels (und somit des Endes) zu hören (dergestalt, dass man an dem vorherigen Zustand eigentlich noch festhalten oder in ihm noch verweilen wollte). Die Erweiterungen sind somit ein Mittel der Differenzierung und wohl auch Dramatisierung, und damit letztlich auch Poetisierung der Musik.

\section{Übergang zur Reprise}

Im Unterschied zur Syntax der Hauptthemen, die auf klar definierten Modellen hinsichtlich Länge, Phrasenbildung und Harmonik basieren, ist die Gestaltung des Übergangs zur Reprise weniger standardisiert bzw. durch Normen vorgegeben. Festhalten lässt sich allerdings, dass die wohl spätestens seit Beethoven für die Sonatenform etablierte Möglichkeit, dem Reprisenbeginn einen Orgelpunkt auf dem Dominantgrundton vorausgehen zu lassen, offenbar auch für Mendelssohn die bevorzugte Option in den Liedern ohne Worte gewesen ist. Nach Agawu kann in einer noch sehr groben Annäherung die Etablierung eines Orgelpunkts als ein Signal oder sstructural sign s gelten, welches anzeigt, dass die Reprise unmittelbar bevorsteht, oder anders gesagt: Der Orgelpunkt ist ein Zeichen, dass der Anfang vom Ende (des Mittelteils) nun erreicht ist. Angesichts eines derartigen Standards, mithin der Kenntnis eines spezifischen Erwartungshorizonts, lassen sich in den Liedern ohne Worte nun unterschiedliche und zum Teil entgegengesetzte Strategien der Abweichung von diesem Standard finden (es gibt natürlich auch dessen Erfüllung). Drei Möglichkeiten lassen sich dabei im Wesentlichen unterscheiden:

- das erreichte Ende wird wieder in Frage gestellt bzw. erst allmählich gefestigt;

- das Ziel (der Reprisenbeginn) wird durch raschen, plötzlichen Umschwung erreicht;

- der Reprisenbeginn ist in eine größere, die Formteile B und $A^{\prime}$ übergreifende Kadenzbewegung eingebettet.

Die erste Möglichkeit soll an zwei Beispielen demonstriert werden. Hingegen soll die zweite Möglichkeit - da hierüber schon ausführlicher an anderer Stelle publiziert wurde $^{37}$ - nur knapp an einem Beispiel veranschaulicht werden. Die dritte Möglichkeit soll in einem eigenen Abschnitt im Zusammenhang mit der Themenreprise diskutiert werden.

37 Vgl. Scheideler 2014. 
Wie Mendelssohn den Anfang vom Ende zunächst andeutet, dann aber wieder in Frage stellt, lässt sich an Opus 19/1 (MWV U 86; vgl. Bsp. 7) beobachten. Charakteristisches Merkmal des in Takt 16 beginnenden Mittelteils ist ein Gang nach G-Dur, also in eine harmonische Region, die sich vom H-Dur des Endes des A-Teils recht weit entfernt. Für die Rückkehr zur Reprise, die in Takt 29 einsetzt, wird die Musik tonartlich zunehmend in der Schwebe gehalten: Es bleibt eine kurze Zeit unklar, ob noch G-Dur oder bereits wieder e-Moll/E-Dur das tonale Zentrum ist, und somit auch, ob das Ende dieses Mittelteils schon erreicht ist oder noch nicht. Dafür nutzt Mendelssohn die Mehrdeutigkeit eines Akkords aus: Der Akkord c/e/a, der am Ende von Takt 23 erklingt, kann entweder C-Dur mit Sexte (und damit Subdominante von G-Dur) oder aber a-Moll mit Terz im Bass (und damit ebenfalls Subdominante, nun aber von e-Moll/E-Dur) sein. Diese Doppeldeutigkeit wird dazu verwendet, um in Takt 24 G-Dur zu verlassen und recht überraschend den H-Dur-Akkord, also die Dominante von e-Moll/E-Dur, erklingen zu lassen. Aber als ob die Musik sich gleichsam verirrt habe oder vom eingeschlagenen Weg selbst überrascht sei, hält sie einen Augenblick inne - der Melodieton $h^{1}$ wird nicht weitergeführt, sondern bleibt liegen. Die Taktgruppe springt zurück (T. 25 entspricht T. 23), und es kommt zu einem zweiten Anlauf, der allerdings erneut in den H-DurAkkord mündet. An diesem Punkt bleibt die Musik erneut gleichsam stehen. Der Orgelpunkt ist zwar Zeichen für die unmittelbar bevorstehende Reprise, aber der Stillstand der Musik erweckt (nach dem vorausgegangenen Changieren zwischen G-Dur und e-Moll/E-Dur) doch zugleich den Eindruck des Zögerns oder einer Unentschlossenheit (melodisch auch durch das Umkreisen des Tons $h^{1}$ durch ais ${ }^{1}$ und $c^{1}$ in den Takten 26-28 deutlich gemacht). Erst tastend, dann aber in umso hellerem E-Dur wird der Eintritt der Themenreprise vollzogen.
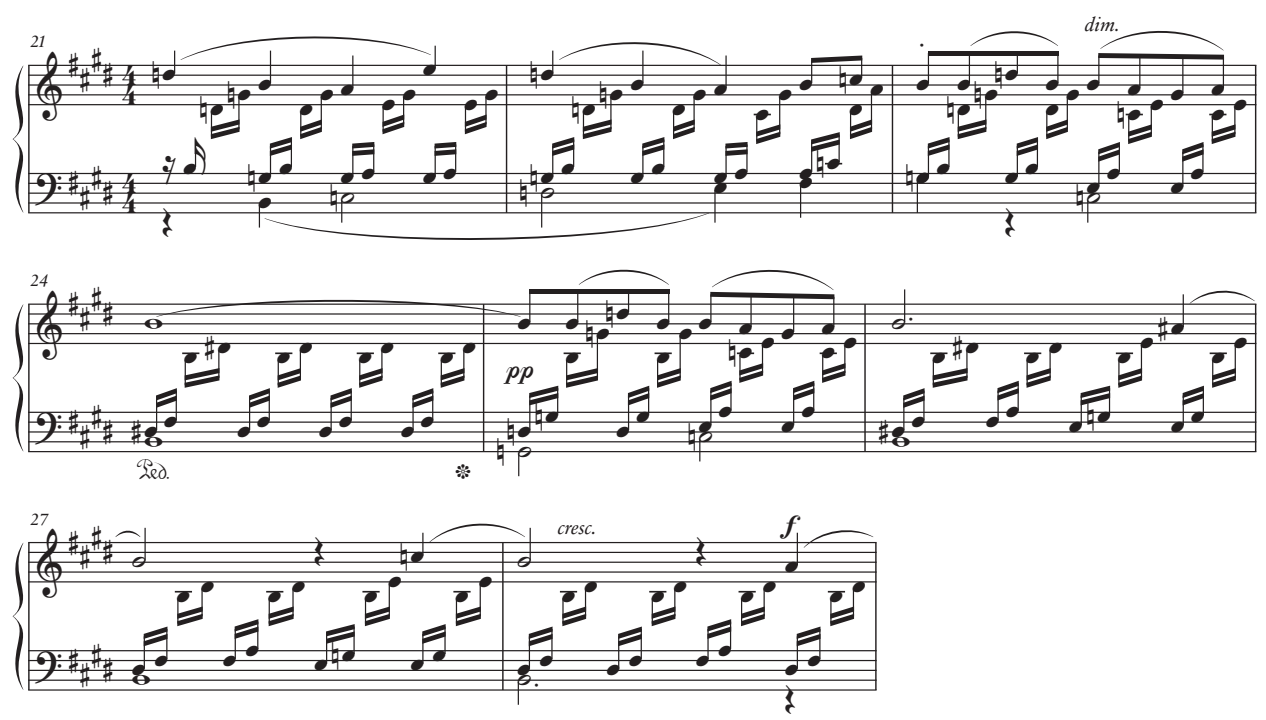

Beispiel 7: Felix Mendelssohn Bartholdy, Lied ohne Worte op. 19/1 (MWV U 86), T. 21-28 
Was sich hier im Kleinen zeigt, hat Mendelssohn in anderen Stücken ausgebaut. ${ }^{38}$ Stellvertretend für eine Reihe weiterer Beispiele sei hier das Lied ohne Worte op. 67/1 (MWV U 180; vgl. Bsp. 8) analysiert, in dem der Weg in die Reprise ebenfalls von einem harmonischen Changieren geprägt ist, wenn auch auf andere Weise. Das in Es-Dur stehende Stück geht im Mittelteil harmonisch zunächst in Terzen aufwärts: erst (ab T. 11) nach g-Moll, dann nach B-Dur (T. 14). B-Dur scheint dann (als Dominante zum tonikalen Es-Dur) die Rückkehr einzuleiten (T. 15), ist jedoch Ausgangspunkt für eine Ausweichung nach Ges-Dur (T. 18), das sich allerdings nur als kurze Zwischenstufe entpuppt. Die in Takt 18 erklingende Subdominante Ces-Dur mit Sexte wird erneut als as-Moll mit Terz im Bass umgedeutet, wodurch wieder der Bezug (als Subdominante) zu Es-Dur hergestellt wird (durch den Ton a am Ende von T. 18 und somit die doppeldominantische Einfärbung wird der Es-Dur-Bezug weiter gestärkt). Das sich anschließende B-Dur auf schwerer Zeit scheint diese Deutung zu bestätigen, da es im Kontext eines phrygischen Halbschlusses erklingt (die zweite Hälfte von T. 23 könnte nahtlos an die erste Hälfte von T. 19 anschließen). Auch die Wiederholung der zweiten Hälfte von Takt 18 in Takt 19 deutet noch eine Bestätigung an, erweist sich aber als das genaue Gegenteil, nämlich als erster Schritt weg vom Halbschluss: Statt im Bass von ces in Takt 20 zu B zurückzukehren, geht der Bass chromatisch aufwärts. Die steigende Sequenz bringt ein Moment der Emphase oder des Ausbruchs ins Spiel, zugleich auch eine ziellose harmonische Bewegung, deren Ende prinzipiell unvorhersehbar ist. Sie endet hier zwar auf dem Ton es (T. 21, Zählzeit 3), doch ist dieser nicht Grundton der Tonika, sondern Septime der ii. Stufe. Dann aber verebbt der Ausbruch, sodass der Wiedereintritt des Hauptthemas als Reprise in Takt 23 gleichsam als ein Moment der Entspannung nach einer Phase der Unruhe (oder gar Gefährdung) inszeniert werden kann.

Wurde die Rückkehr zum Ausganspunkt hier gleichsam aufgeschoben oder verzögert, so lassen sich auch gegenteilige Tendenzen finden: im plötzlichen und unerwarteten Auftreten des Anfangsthemas. Ein Beispiel für diese Inszenierungsweise stellt das Lied ohne Worte op. 67/3 (MWVU 102; vgl. Bsp. 9) dar. ${ }^{39}$ Hier hat Mendelssohn einen Satz geschrieben, der sowohl von einer gewissen Unruhe durchzogen (aufgrund der pochenden Synkopen) als auch (im ersten Teil) von einem Idyllen- und Naturton geprägt ist, der u.a. durch die Tonart B-Dur, den pentatonischen Beginn der Melodie mit dem Tonvorrat $b-c-d-f-g$, die strenge Diatonik der Takte 1-8 sowie die Betonung der Subdominante hervorgerufen wird. Der Mittelteil, der durch seine Chromatik einen deutlichen Kontrast bildet, verebbt schließlich über dem dominantischen Orgelpunkt a. Dieser Orgelpunkt ist zwar wieder ein klares Reprisenvorbereitungssignal, doch ist gleichzeitig auch hörbar, dass nicht die Haupttonart B-Dur, sondern d-Moll vorbereitet wird. An diesem Punkt im musikalischen Verlauf kommt der Satz (wie schon in Opus 19/1) gewissermaßen zum Stillstand bzw. gerät in eine Sackgasse: harmonisch durch das Verharren

38 Vgl. in ähnlicher Weise etwa das Gondellied fis-Moll op. 30/6 (MWVU 110) oder das Lied ohne Worte E-Dur op. 38/3 (MWV U 107).

39 Vgl. hierzu ausführlicher Scheideler 2014, 7-12, wo nicht nur diese Takte analysiert werden, sondern weitere Beispiele (MWV U 89, 97, 114, 154, 181, 190) für die Möglichkeit, die Reprise als unvorhersehbaren Punkt im Formverlauf bzw. plötzliche Rückkehr zu inszenieren, diskutiert werden. 


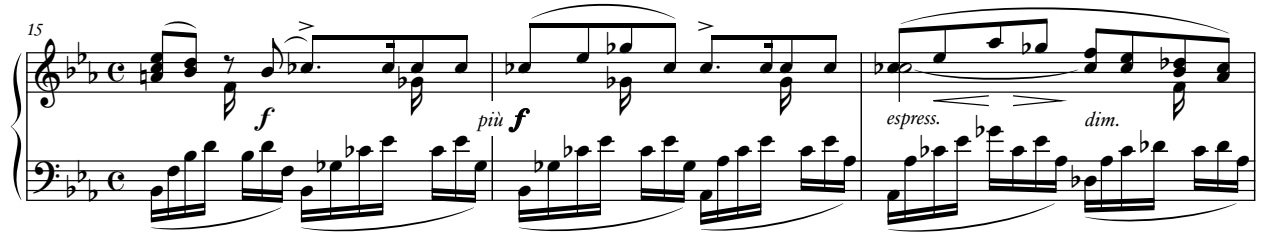

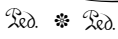

* S 2 d.

* ido. *
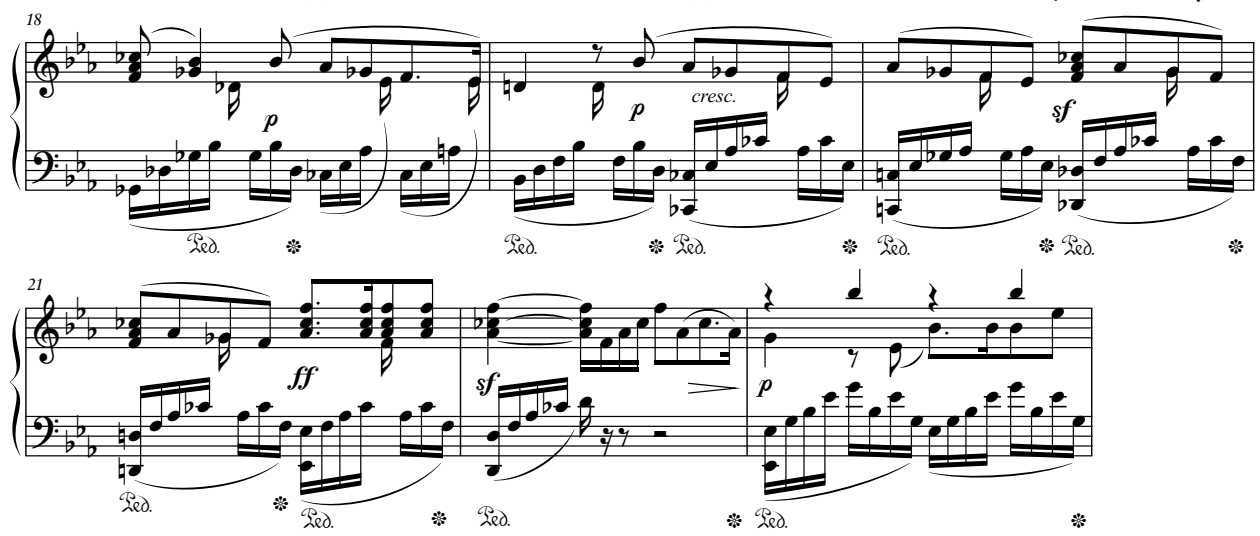

Beispiel 8: Felix Mendelssohn Bartholdy, Lied ohne Worte op. 67/1 (MWV U 180), T. 15-23

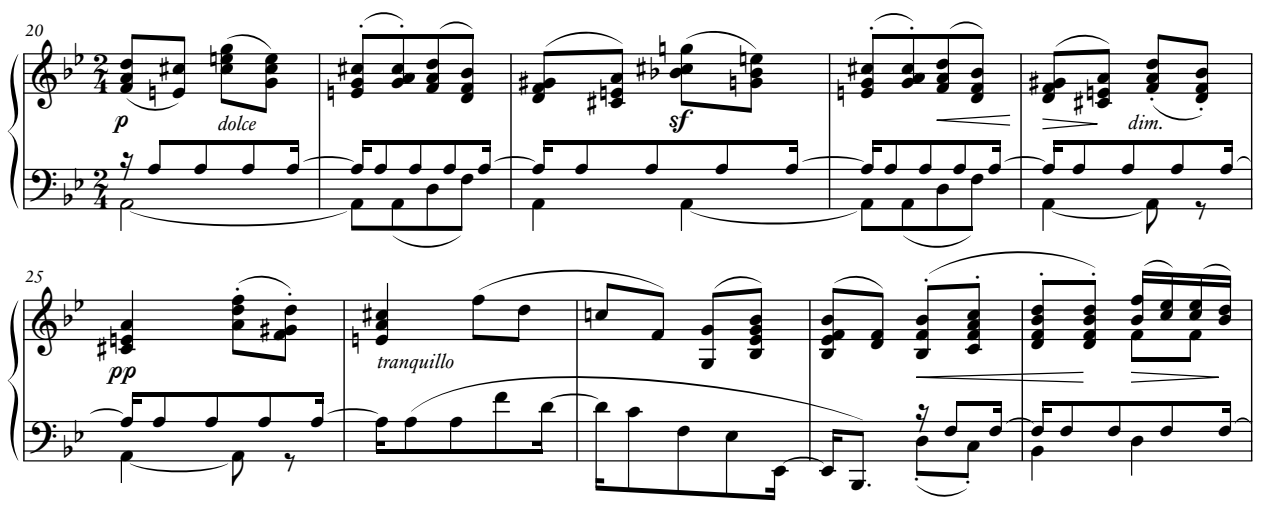

Beispiel 9: Felix Mendelssohn Bartholdy, Lied ohne Worte op. 67/3 (MWV U 102), T. 20-29

auf dem Orgelpunkt, motivisch durch die allmähliche Auflösung der Viertonfigur, die ab der Mitte von Takt 23 wiederholt und dann ihres 'Seufzers` beraubt wird (vgl. T. 24 mit T. 25), sodass eine Viertelnote den Abschluss bildet. Nun wird die Figur sequenziert (T. 25/26) - und dann brechen wie aus dem Nichts plötzlich das Hauptthema und somit die B-Dur-Sphäre in den musikalischen Verlauf ein. Kompositorisch ist das durchaus einfach gemacht, denn die Töne $f^{2}-d^{2}$ von Takt 26 (hier ohne akkordische Auffüllung, 
sodass für einen Augenblick die Tonalität offen bleibt) wiederholen die bereits in Takt 25 erklingenden Töne, die hier noch auf d-Moll bezogen sind. Aber die Wirkung ist doch eine ganz außerordentliche: Die Stelle hat etwas Befreiendes, sie ließe sich daher (vielleicht) als Einbruch des Wunderbaren oder als Moment der Erlösung interpretieren.

\section{Themenreprise}

Neben der Möglichkeit, den A'-Teil einer dreiteiligen Liedform als eine vollständige oder gar (beträchtlich) erweiterte Reprise zu komponieren, gab es seit der zweiten Hälfte des 18. Jahrhunderts stets die Möglichkeit, den A'-Teil als komprimierte Variante des A-Teils aufzufassen (so im klassischen Tanzmenuett mit den Proportionen $8+4+4$ Takte). Dass der A'-Teil kürzer ist als der A-Teil, ist auch in Mendelssohns Liedern ohne Worte die Regel. Wichtiger für die Form des A'-Teils ist jedoch, dass das Thema oft nicht mehr vollständig erklingt, sondern lediglich dessen Vordersatz, während der Nachsatz nur noch Teilmomente aufgreift und neu fortspinnt (wodurch der gesamte $A^{\prime}$-Teil auch länger als der A-Teil ausfallen kann). Ganz wesentlich ist in diesem Zusammenhang die Harmonik, und hier vor allem die Setzung der Tonika. Mehrfach lässt sich nämlich beobachten, wie zum einen Mittelteil und $A^{\prime}$-Teil als Teil einer großen Kadenzbewegung komponiert sind, und zum anderen eine starke Tonika im A'-Teil bis zum Ende aufgespart wird. Dadurch aber wird die Auffassung der Form verändert: Das entscheidende Ereignis im Formverlauf ist nicht mehr so sehr der Wiedereintritt des Themas am Beginn des $A^{\prime}$-Teils bzw. die Themenreprise (da sie nicht mit dem Wiedereintritt der Tonika zusammenfällt) als vielmehr das Ende des A'-Teils, an dem die Tonika nach einer emphatischen Bewegung endlich wieder erreicht ist. Zwei Werkausschnitte sollen die Inszenierungsstrategien veranschaulichen. ${ }^{40}$ Nummer 1 (MWV U 185; vgl. Bsp. 10) aus der Sammlung Opus 62, die 1844 erschien und Clara Schumann gewidmet ist, hat eine konventionelle dreiteilige Form (A: T. 1-10, B: T. 11-22, A': T. 22-35) mit einer kurzen Coda. Das Stück ist insofern recht gleichförmig, als das rhythmisch-intervallische Modell der Begleitung stets beibehalten wird und der Melodik nur drei Motive zugrundeliegen: ein Sprung abwärts mit zwei Vierteln (auftaktiger Beginn), ein Achtelmotiv mit einer bogenförmigen Anlage sowie ein abschließendes sSeufzermotiv (T. 1/2 mit Auftakt; identisch in T. 22-24; vgl. Bsp. 10). Die Beschränkung auf nur wenige Motive und Spielfiguren wird ausgeglichen durch einen Variantenreichtum, der einen steten Wechsel der Formung der Figuren zur Folge hat. Eine Besonderheit besteht zudem darin, dass das Stück mit der Dominante statt der Tonika beginnt. Das muss notwendigerweise Konsequenzen für den Übergang zur Reprise haben, denn es ist jetzt nicht mehr (oder nur schlecht) möglich, an das Ende des Mittelteils einen dominantischen Orgelpunkt zu setzen. In der Tat hat Mendelssohn sich für einen anderen Weg entschieden, der weitreichende Folgen für die tonale Gliederung des gesamten Stückes hat.

Nachdem der erste Teil mittels Verdoppelung der Kadenz auf zehn Takte gestreckt wurde und in h-Moll endet, beginnt der Mittelteil in e-Moll (T. 11), um mittels einer Steigerung über chromatischem Bass in Takt 15 einen G-Dur-Septakkord zu erreichen.

40 MWV U 185 wird kurz besprochen auch in Scheideler 2014, 3. 

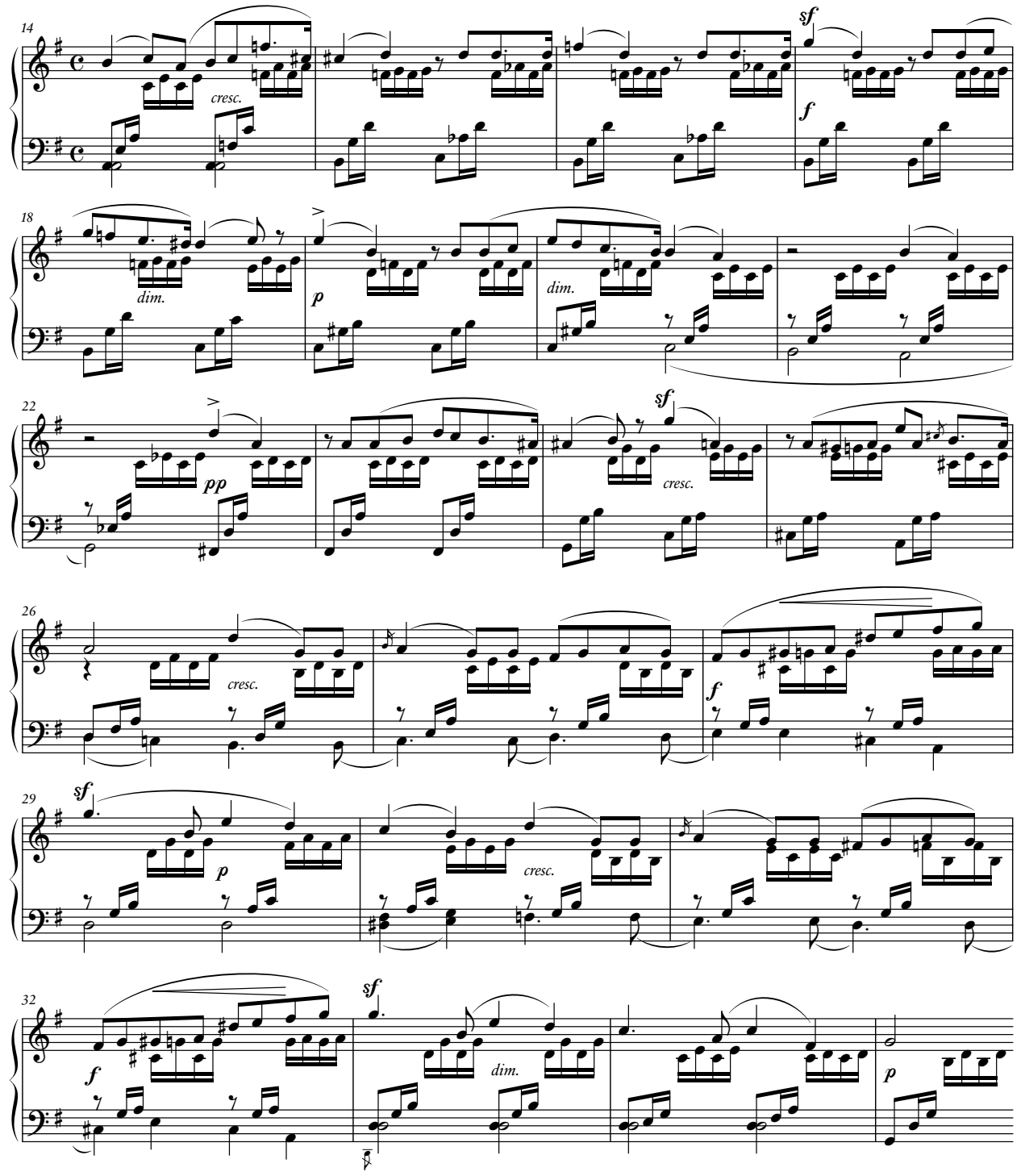

Beispiel 10: Felix Mendelssohn Bartholdy, Lied ohne Worte op. 62/1 (MWV U 185), T. 14-35

Der Übergang zu C-Dur wird indes vorerst nicht vollzogen, vielmehr kommt es zu einer Pendelbewegung zwischen G-Dur-Septakkord und f-Moll mit Quinte und Sexte, oder, funktional betrachtet, zwischen Dominante und Prädominante (bezogen auf C-Dur). Es ist, als müsse die Melodie gleichsam erst Anlauf nehmen, um den Schritt des Übergangs in die lokale Tonika tatsächlich zu vollziehen, was erst in Takt 18 geschieht, nachdem das zweite Motiv von seiner Dreitonfigur (vgl. T. 15 und 16) wieder auf sieben Töne erweitert 
wurde. Das Crescendo ab Takt 14, das in Takt 17 endet, macht deutlich, dass hier eine Reprise (bzw. Scheinreprise) angedeutet ist, die nicht nur in der falschen Tonart (C-Dur), sondern auch an metrisch geänderter Position steht (um einen halben Takt versetzt). In dem Moment, in dem der Basston c (zugleich mit der lokalen Tonika C-Dur) eintritt (T. 18), wird an ihm zwar endgültig festgehalten, darüber erklingt dann aber E-Dur mit Septime und None als Dominante zu a-Moll, das in Takt 20 erreicht ist. Danach setzt im Bass eine lineare Bewegung zu Fis ein, die mit einer Abspaltung des Schlussmotivs von Takt 20 im Diskant verbunden ist, aus dem dann die melodische Reprise erwächst. Am Ende des Mittelteils steht hier also kein dominantischer, sondern ein prädominantischer Orgelpunkt (ab T. 18) bzw. eine Prolongation der Prädominante (ab T. 15). Der Reprisenbeginn fällt dann nicht mit dem Übergang zur Tonika, sondern mit dem zur Dominante zusammen. Daraus zieht nun Mendelssohn eine weitere Konsequenz, denn der Eintritt einer starken Tonika wird mehrfach hinausgeschoben. Wie schon im A-Teil erklingt eine schwache Tonika nur in Takt 24 (mit Vorhalt und dann in Terzlage), danach tritt sie (als Sextakkord) in Takt 26 auf. Erreicht wird sie schließlich erst in Takt 35. Mendelssohn inszeniert diesen besonderen Punkt, die endlich am Ziel angelangte Bewegung, wieder durch Prolongation der Kadenzstationen, durch Verdoppelung der Kadenz (ein Ganzschluss wäre erstmals in T. 30 zu erwarten, wird jedoch trugschlüssig vermieden), vor allem aber durch eine Melodik, in der jetzt das zweite Motiv fortgesponnen wird (vgl. T. 27/28 und T. 31/32). Man kann hier wohl so weit gehen zu sagen, dass das gesamte Stück auf diesen Moment des Eintritts der Tonika hinzielt - denn es gibt vorher keine einzige Stelle, an der G-Dur als starke Tonika hörbar ist. Vielmehr lässt der gesamte harmonische Verlauf folgende Stationen als wesentlich erscheinen:

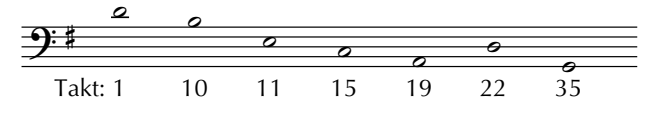

Beispiel 11: Felix Mendelssohn Bartholdy, Lied ohne Worte op. 62/1 (MWV U 185), übergeordneter harmonischer Verlauf (in Grundtönen)

Die Übersicht zeigt, dass es übergeordnet einen durchgängigen Terzfall gibt, der jedoch am Anfang an einer Stelle gleichsam übersprungen wird, nämlich genau dort, wo G-Dur hätte erklingen müssen. G-Dur ist erst am Ende deutlich hervorgekehrte Tonika, die durch zwei vorangehende Quintfälle akzentuiert wird. Damit werden alle drei Formteile durch den übergeordneten harmonischen Verlauf zu einer Einheit zusammengebunden. Der Reprisenbeginn ist dabei nicht das primäre Ziel, sondern stellt eine Zwischenstation oder gar einen Anfang dar. Das Hinauszögern des Reprisenbeginns bewirkt, dass der $A^{\prime}$-Teil wie eine Befreiung empfunden werden kann, eine Wirkung, die durch den zweimal gespielten Spitzenton $g^{2}$ (T. 29,33) noch unterstützt wird. Der Aufschwung im A'-Teil ist jedoch nicht allein die unmittelbare Folge der Stauung der Bewegung am Ende des B-Teils, sondern eben auch der zahlreichen Verlängerungen, die das Ende immer weiter hinausschieben. Die unterschiedlichen Arten der Zeitgestaltung erscheinen somit unmittelbar aufeinander bezogen.

Eine vergleichbare Strategie lässt sich im 1845 entstandenen, aber erst postum publizierten Lied ohne Worte op. 85/4 (MWV U 190; vgl. Bsp. 12) beobachten. Hier be- 
ginnt die Reprise in Takt 20 auf dem Tonikagegenklang fis-Moll. Der Nachsatz (T. 24 ff.) umkreist die Tonika D-Dur mittels schwebender Tonalität, erreicht diesen Akkord selbst als emphatische Tonika aber erst in Takt 32. Auch hier finden sich innere Erweiterung (T. 24-27) und Verdoppelung der Kadenz (die starke Dominante in T. 28 wird in einen Sextakkord der Tonika überführt). Hinzu kommt die harmonische Abschweifung in Takt 27 und 30, die den musikalischen Satz kurz in Richtung h-Moll lenkt, ohne dort jedoch jemals anzukommen (nach Doppeldominante Cis-Dur tritt zwar die Dominante Fis-Dur ein, doch wird der Quartsextvorhalt in Takt 28 und Takt 31 nicht regelgerecht aufgelöst, sondern zu einem e-Moll-Akkord weitergeführt, sodass sich A-Dur und damit die Dominante zum tonikalen D-Dur anschließen kann).

Nach verhaltenem Beginn schwingt sich der Satz hier mehrfach mit großer Emphase auf. Und auch hier erweckt die Musik (d. h.: der Eintritt der Zieltonika) den Eindruck, als wolle sie an gar kein Ende gelangen, als wolle sie den Zustand der Erfüllung immer weiter hinauszögern, bis das Erreichen des Ziels sich nicht länger aufschieben lässt.
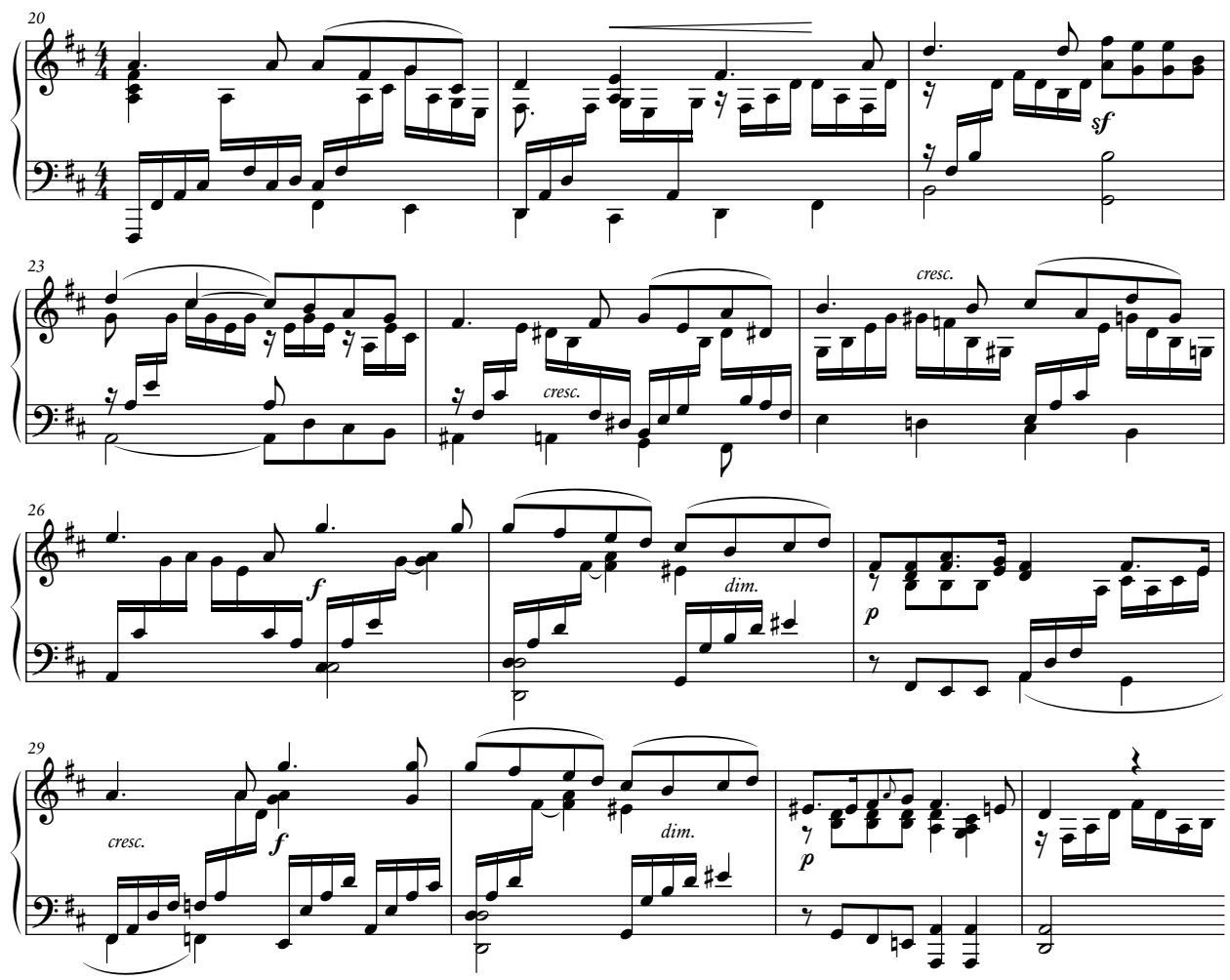

Beispiel 12: Felix Mendelssohn Bartholdy, Lied ohne Worte op. 85/4 (MWV U 190), T. 20-32

Die Themenreprise, so haben die vorangehenden Analysen zu zeigen versucht, wird in den Liedern ohne Worte oft als ein ganz besonderer Punkt im Werkverlauf herausge- 
stellt. Obschon die Art der Inszenierung ihn deutlich markiert und heraushebt, haftet ihm gleichwohl etwas Vorläufiges an. Das lässt sich auch daran ablesen, dass die Reprise in der überwiegenden Anzahl der Fälle im Piano und ganz verhalten beginnt. ${ }^{41}$ Die Wiederkehr des Hauptthemas wird also deutlich als eine bloß vorläufige Station kenntlich gemacht, die wiederum zum Ausgangspunkt einer Entwicklung wird, die erst am Ende des A'-Teils ihr Ziel (und gleichsam ihre Erfüllung) findet. Die Gewichte im Formprozess haben sich somit verschoben. Nicht der Anfang vom Ende, sondern das Ende vom Ende wird als herausgehobener Punkt (und Ziel) im Formverlauf inszeniert. Oft wird schon frühzeitig im Stück durch verschiedene Maßnahmen das bevorstehende Ende hörbar, sodass sich auf der einen Seite eine linear aus- und zielgerichtete Entwicklung feststellen lässt, die andererseits - wie gezeigt - nicht gleichmäßig verläuft, sondern retardierende wie beschleunigende Momente enthält. Das Ende tritt schließlich als ein endlich erreichtes (oder ersehntes) Ziel ein, auf das hin der gesamte Formprozess ausgerichtet war. Eine derartige Dramaturgie aber ist das Ergebnis des Zusammenspiels von Motivik, Harmonik und Syntax.

III

Mendelssohn hat in seinem berühmten Brief an Marc-André Souchay vom 15. Oktober 1842, in welchem er zu dem Vorschlag Stellung nahm, die Lieder ohne Worte mit Worten zu unterlegen, geschrieben: „Die Leute beklagen sich gewöhnlich, die Musik sei so vieldeutig; es sei so zweifelhaft, was sie sich dabei zu denken hätten, und die Worte verstände doch ein Jeder. Mir geht es aber gerade umgekehrt. [...] Das, was mir die Musik ausspricht, die ich liebe, sind mir nicht zu unbestimmte Gedanken, um sie in Worte zu fassen, sondern zu bestimmte. [...] Fragen Sie mich, was ich mir dabei gedacht habe, so sage ich: gerade das Lied wie es dasteht [...], weil nur das Lied dem Einen dasselbe sagen, dasselbe Gefühl in ihm erwecken kann, wie im Andern. - ein Gefühl, das sich aber nicht durch dieselben Worte ausspricht «. ${ }^{42}$ Den Liedern ohne Worte liegen also gemäß dem Zeugnis des Komponisten Gedanken, Gefühle oder Gehalte zugrunde, nur lassen sich diese nicht allgemeingültig verbal benennen. ${ }^{43}$ Die Sprachskepsis zeigt eine Nähe zum romantischen Unsagbarkeitstopos, hier ist Mendelssohns Denken ganz romantischen Ideen verpflichtet. Das Unsagbare wird durch die Musik fühlbar, doch erscheint eine (konkrete) Semantisierung (auch nur einzelner Stellen) kaum möglich. In der Tat liegen, von wenigen Ausnahmen abgesehen, keine sreferential signs im Sinne Agawus vor. Der deutliche Bezug auf Jagdidiomatik in op. 19/3 (MWV U 89) oder auf einen Trauermarsch op. 62/3 (MWV U 177) stellen Ausnahmen dar, und selbst die drei Gondellieder geben eigentlich nicht viel mehr als einen vagen Assoziationsrahmen vor,

41 Ausnahmen stellen u.a. die Lieder ohne Worte op. 19/1 (MWVU 86), op. 19/2 (MWV U 80), op. 38/2 (MWV U 115), op. 53/6 (MWVU 154) sowie op. 62/6 (MWVU 161) dar.

42 Zitiert nach Schmidt 1996, 155.

43 Mendelssohn hatte freilich nichts gegen eine Textunterlegung, so lange die Verse nicht beanspruchten, über eine individuelle Umschreibung der Gedanken und Gefühle hinauszugehen (vgl. Schmidt 1996, 286). 
der sich nicht mit konkreten Bildern füllen lässt. ${ }^{44}$ Umgekehrt bleibt aber auch klar, dass es sich bei dieser Musik weder um absolute Musik noch um ein Spiel mit Versatzstücken (wie bei Haydn) oder auch um ein ausschließliches Komponieren mit der Form (Dahlhaus ${ }^{45}$ ) handelt. Nicht nur der Titel »Lied ohne Worte«, sondern auch die Musik selbst drückt aus, dass hier das Reich der Poesie das Zentrum der Gattung bildet. Die von Thomas Christian Schmidt zusammengetragenen verstreuten Äußerungen Mendelssohns bestätigen diese ästhetischen Prämissen. ${ }^{46}$

Worin aber liegt der damit angedeutete spoetische` Ton begründet? Ließe sich mit Bezug auf die von Moßburger apostrophierte spoetischer Harmonik Schumanns im Fall von Mendelssohn analog von einer spoetischen Syntax sprechen? Die technischen Verfahren der Erweiterungen, der Verzögerung und Beschleunigung lassen sich mit den Werkzeugen der älteren wie neuen Musiktheorie recht gut beschreiben. Das macht deutlich, dass Mendelssohn sowohl die klassischen Formen als auch die schon im 18. Jahrhundert geschilderten Umgangsweisen mit diesen aufruft, ihnen aber offensichtlich einen neuen Sinn gibt, sodass nicht durch eine avancierte Harmonik oder besondere Spielfiguren, sondern durch eine Umwertung oder Umdeutung klassischer Verfahrensweisen ein spoetischer Ton hervorgebracht wird. Damit ließe sich auch die häufig diskutierte Frage, ob Mendelssohn Klassizist oder Romantiker sei, vielleicht so auflösen: Das klassische Werkzeug wird weiter verwendet, aber in neuer Funktion: Nicht also die Technik und das Material, sondern ihr Gebrauch ist somit entscheidend. Die nuancierte Zeitgestaltung auf kleinstem Raum bringt gleichsam jenes Ineinander von `Ahnung und Gegenwart hervor, das seit Eichendorff als ein Inbegriff des Poetischen und somit Romantischen gelten kann. Und dieser Gebrauch ist ganz entscheidend von der Idee bestimmt, durch die Erweiterungen bzw. das Aufbrechen der regelmäßigen Viertakt- und Achttaktgruppen das Statische oder Architektonische der kleinen musikalischen Form zu überwinden, indem Übergänge als Zögern oder plötzlicher Einbruch inszeniert werden oder aber ein schon angedeutetes Ende hinausgeschoben und dadurch in seiner Bedeutung aufgeladen wird. Die sprosaische` Zeit, jene bloß gleichmäßig abrollende oder dahinfließende Zeit, wird somit gleichsam poetisiert. Vor diesem Hintergrund wäre es dann weniger die liedhafte Melodik als vielmehr der besondere Umgang mit Syntax und Form ${ }^{47}$, der das Lied ohne Worte zu einer romantischen Gattung par excellence machen würde.

44 Knappe Analysen, die aber auf die Frage nach der Beziehung von Titel und Musik nur am Rand eingehen, finden sich bei Jost 1988, 153 ff., sowie bei de la Motte 1968, 125-130 (zum Gondellied fis-Moll op. 30/6).

45 Vgl. Dahlhaus 1987, insbesondere $207 \mathrm{ff}$.

46 Vgl. hierzu Schmidt 1996, insbesondere 285-300.

47 In diesem Zusammenhang wären auch die Einleitungen in vielen Liedern ohne Worte genauer zu analysieren, die den Zuhörer zunächst gleichsam aus der sprosaischen` Welt hinausführen, ehe die spoetischer Welt beginnt. 


\section{Literatur}

Agawu, Kofi (1991), Playing with Signs. A Semiotic Interpretation of Classic Music, Princeton: Princeton University Press.

(2009), Music as Discourse. Semiotic Adventures in Romantic Music, Oxford: Oxford University Press.

Dahlhaus, Carl (1987), Beethoven und seine Zeit, Laaber: Laaber.

de la Motte, Diether (1968), Musikalische Analyse, mit kritischen Anmerkungen von Carl Dahlhaus, Kassel u. a.: Bärenreiter.

Jost, Christa (1988), Mendelssohns Lieder ohne Worte, Tutzing: Hans Schneider.

Koch, Heinrich Christoph (1793), Versuch einer Anleitung zur Composition, Bd. 3, Leipzig: Adam Friedrich Böhme, Reprint in: ders., Versuch einer Anleitung zur Composition, hg. von Jo Wilhelm Siebert, Hannover: Siebert 2007, 405-572.

Marx, Adolf Bernhard (1864), Die Lehre von der musikalischen Komposition, praktischtheoretisch, Bd. 2, 5. verbesserte Ausgabe, Leipzig: Breitkopf \& Härtel.

Moßburger, Hubert (2005), Poetische Harmonik in der Musik Robert Schumanns (= Musik und Musikanschauung im 19. Jahrhundert 10), Sinzig: Studio.

Riepel, Joseph (1996), Grundregeln zur Tonordnung insgemein (1755), Frankfurt a.M. und Leipzig, Reprint in: Joseph Riepel, Sämtliche Schriften zur Musiktheorie, hg. von Thomas Emmerig, Wien u. a.: Böhlau, 103-237.

Scheideler, Ullrich (2014), „Der Übergang zur Reprise in Mendelssohns /Liedern ohne Worte«. https://www.udk-berlin.de/fileadmin/2_dezentral/FR_Musiktheorie/kPortal/ kportal_scheideler_liederohneworte.pdf

Schmidt, Thomas Christian (1996), Die ästhetischen Grundlagen der Instrumentalmusik Felix Mendelssohn Bartholdys, Stuttgart: Metzler und Poeschel. 